\title{
PICTURE FUZZY CROSS-ENTROPY FOR MULTIPLE ATTRIBUTE DECISION MAKING PROBLEMS
}

\author{
Guiwu WEI \\ School of Business, Sichuan Normal University, Chengdu, 610101, P.R. China \\ E-mail:weiguiwu@163.com
}

Received 18 April 2016; accepted 31 May 2016

\begin{abstract}
In this paper, we investigate the multiple attribute decision making problems with picture fuzzy information. The advantage of picture fuzzy set is easily reflecting the ambiguous nature of subjective judgments because the picture fuzzy sets are suitable for capturing imprecise, uncertain, and inconsistent information in the multiple attribute decision making analysis. Thus, the cross entropy of picture fuzzy sets, called picture fuzzy cross entropy, is proposed as an extension of the cross entropy of fuzzy sets. Then, a multiple attribute decision making method based on the proposed picture fuzzy cross entropy is established in which attribute values for alternatives are picture fuzzy numbers. In decision making process, we utilize the picture fuzzy weighted cross entropy between the ideal alternative and an alternative to rank the alternatives corresponding to the cross entropy values and to select the most desirable one(s). Finally, a practical example for enterprise resource planning system selection is given to verify the developed approach and to demonstrate its practicality and effectiveness.
\end{abstract}

Keywords: multiple attribute decision making, fuzzy set, picture fuzzy set, picture fuzzy cross-entropy, picture fuzzy weighted cross-entropy, enterprise resource planning, system selection.

JEL Classification: C61.

\section{Introduction}

Entropy is very important and effective tool for measuring uncertain information. Firstly, Zadeh $(1965,1968)$ introduced the fuzzy entropy. The starting point for the crossentropy approach is information theory as developed by Shannon (1948). Kullback and Leibler (1951) proposed the "cross-entropy distance" measure between two probability distributions. Later, Lin (1991) proposed a modified cross-entropy measure. Shang and Jiang (1997) proposed a fuzzy cross-entropy measure and a symmetric discrimination information measure between fuzzy sets. Vlachos and Sergiadis (2007) developed the intuitionistic fuzzy cross-entropy based on the De Luca-Termini non-probabilistic entropy (Deluca, Fernuni 1972). Zhang and Jiang (2008) defined the cross-entropy between vague sets. According to the cross-entropy of vague sets, Ye (2009a) has investigated the fault diagnosis problem of turbine. Ye (2009b) has applied the intuitionistic fuzzy cross- 
entropy to multicriteria fuzzy decision-making problems. Ye (2011) proposed an intervalvalued intuitionistic fuzzy cross-entropy for multiple attribute decision making problems on the basis of the vague cross-entropy. Xia and $\mathrm{Xu}$ (2012) proposed some cross-entropy and entropy formulas for intuitionstic fuzzy sets and applied them to group decisionmaking. For interval-valued intuitionistic fuzzy sets, Zhang and Jiang (2010) proposed the entropy and cross-entropy concepts and discussed the connections among some important information measures. Xu and Xia (2012) introduced the concepts of entropy and cross-entropy for hesitant fuzzy information, and discuss their desirable properties.

Recently, Cuong (2013) proposed picture fuzzy set (PFS) and investigated the some basic operations and properties of PFS. The picture fuzzy set is characterized by three functions expressing the degree of membership, the degree of neutral membership and the degree of non-membership. The only constraint is that the sum of the three degrees must not exceed 1. Basically, PFS based models can be applied to situations requiring human opinions involving more answers of types: yes, abstain, no, refusal, which can't be accurately expressed in the traditional FS and IFS. Until now, some progress has been made in the research of the PFS theory. Singh (2014) investigated the correlation coefficients for picture fuzzy set and apply the correlation coefficient to clustering analysis with picture fuzzy information. Son (2015) and Thong and Son (2015) introduced several novel fuzzy clustering algorithms on the basis of picture fuzzy sets and applications to time series forecasting and weather forecasting. Thong (2015) developed a novel hybrid model between picture fuzzy clustering and intuitionistic fuzzy recommender systems for medical diagnosis and application to health care support systems.

Due to the advantage of picture fuzzy set is easily reflecting the ambiguous nature of subjective judgments because the picture fuzzy sets are suitable for capturing imprecise, uncertain, and inconsistent information in actual multiple attribute decision making analysis as mentioned above, it is necessary to develop some cross-entropy measures for picture fuzzy set. In order to do so, the remainder of this paper is set out as follows. In the next section, we introduce some basic concepts related to intuitionistic fuzzy set and picture fuzzy sets. In Section 2, we shall propose the picture fuzzy cross-entropy and picture fuzzy weighted cross-entropy. In Section 3, based on picture fuzzy weighted cross-entropy, we shall present the model for multiple attribute decision making problems with picture fuzzy information. In Section 4, we shall present a numerical example for enterprise resource planning (ERP) system selection with picture fuzzy information in order to illustrate the method proposed in this paper. The last section concludes the paper with some remarks.

\section{Preliminaries}

In the following, we introduce some basic concepts related to intuitionistic fuzzy sets.

Definition 1 (Atanassov 1986, 1989). An IFS $A$ in $X$ is given by:

$$
A=\left\{\left\langle x, \mu_{A}(x), v_{A}(x)\right\rangle \mid x \in X\right\},
$$

where $\mu_{A}: X \rightarrow[0,1]$ and $v_{A}: X \rightarrow[0,1]$, where, $0 \leq \mu_{A}(x)+v_{A}(x) \leq 1, \forall x \in X$. The 
number $\mu_{A}(x)$ and $v_{A}(x)$ represents, respectively, the membership degree and nonmembership degree of the element $x$ to the set $A$.

The intuitionistic fuzzy sets have received more and more attention since its appearance. Kosareva and Krylovas (2013) established the types of intuitionistic fuzzy numbers and the exponent values of the generalized weighted averaging operator having the least error probabilities considering alternatives ranking. Razavi Hajiagha et al. (2013) defined a complex proportional assessment method for group decision making in an interval-valued intuitionistic fuzzy environment. Krohling et al. (2013) also extended the intuitionistic fuzzy TODIM to multi-criteria decision making. Chen (2014) proposed the interval-valued intuitionistic fuzzy QUALIFLEX method with a likelihood-based comparison approach for multiple criteria decision analysis. Lourenzutti and Krohling (2013) developed the TODIM method in a intuitionistic fuzzy and random environment. Wu et al. (2013) researched on the AHP with interval-valued intuitionistic fuzzy sets and its application in multi-criteria decision making problems. Zavadskas et al. (2014) proposed the extension of weighted aggregated sum product assessment with intervalvalued intuitionistic fuzzy numbers. Wang and Liu (2014) developed some hesitant fuzzy geometric operators for multiple attribute group decision making. Razavi Hajiagha et al. (2015) evolved a linear programming technique for MAGDM problems with interval valued intuitionistic fuzzy information. Wu (2015) proposed a SD-IITFOWA operator and TOPSIS based approach for MAGDM problems with intuitionistic trapezoidal fuzzy numbers. Abdullah and Najib (2016) developed a new preference scale MCDM method based on interval-valued intuitionistic fuzzy sets and the analytic hierarchy process. Although, intuitionistic fuzzy set theory (Atanassov 1986, 1989) has been successfully applied in different areas, but there are situations in real life which can't be represented by intuitionistic fuzzy sets. Picture fuzzy sets are extension of intuitionistic fuzzy sets. Picture fuzzy set (Cuong 2013) based models may be adequate in situations when we face human opinions involving more answers of types: yes, abstain, no, refusal. It can be considered as a powerful tool represent the uncertain information in the process of patterns recognition and cluster analysis.

Definition 2 (Cuong 2013). A picture fuzzy set (PFS) A on the universe $X$ is an object of the form:

$$
A=\left\{\left\langle x, \mu_{A}(x), \eta_{A}(x), v_{A}(x)\right\rangle \mid x \in X\right\},
$$

where $\mu_{A}(x) \in[0,1]$ is called the "degree of positive membership of $A$ ", $\eta_{A}(x) \in[0,1]$ is called the "degree of neutral membership of $A$ "and $v_{A}(x) \in[0,1]$ is called the "degree of negative membership of $A$ ", and $\mu_{A}(x), \eta_{A}(x), v_{A}(x)$ satisfy the following condition: $0 \leq \mu_{A}(x)+\eta_{A}(x)+v_{A}(x) \leq 1, \forall x \in X$. Then for $x \in X$, $\pi_{A}(x)=1-\left(\mu_{A}(x)+\eta_{A}(x)+v_{A}(x)\right)$ could be called the degree of refusal membership of $x$ in $A$.

Cuong (2013) also defined some operations as follows.

Definition 3 (Cuong 2013). Given two PFEs represented by $A$ and $B$ on universe $X$, the inclusion, union, intersection and complement operations are defined as follows: 
(1) $A \subseteq B$, if $\mu_{A}(x) \leq \mu_{B}(x), \eta_{A}(x) \leq \eta_{B}(x)$ and $v_{A}(x) \geq v_{B}(x), \forall x \in X$,

(2) $A \cup B=\left\{\left(x, \max \left(\mu_{A}(x), \mu_{B}(x)\right), \min \left(\eta_{A}(x), \eta_{B}(x)\right), \min \left(v_{A}(x), v_{B}(x)\right)\right) \mid x \in X\right\}$,

(3) $A \cap B=\left\{\left(x, \min \left(\mu_{A}(x), \mu_{B}(x)\right), \max \left(\eta_{A}(x), \eta_{B}(x)\right), \max \left(v_{A}(x), v_{B}(x)\right)\right) \mid x \in X\right\}$,

(4) $\bar{A}=\left\{\left(x, v_{A}(x), \eta_{A}(x), \mu_{A}(x)\right) \mid x \in X\right\}$.

For convenience, we call $\alpha=\left(\mu_{\alpha}, \eta_{\alpha}, v_{\alpha}\right)$ a picture fuzzy number (PFN), where:

$$
\mu_{\alpha} \in[0,1], \eta_{\alpha} \in[0,1], v_{\alpha} \in[0,1], \mu_{\alpha}+\eta_{\alpha}+v_{\alpha} \leq 1
$$

\section{Cross-entropy between picture fuzzy sets}

In this section, we shall develop the cross-entropy and discrimination information measures between two PFSs based on the extension of the concept of cross-entropy between two fuzzy sets.

To do this, we firstly introduce the concepts of cross-entropy and symmetric discrimination information measures between two fuzzy sets which were proposed by Shang and Jiang (1997).

Definition 4 (Shang, Jiang 1997). Assume that $\alpha=\left(\alpha\left(x_{1}\right), \alpha\left(x_{2}\right), \cdots, \alpha\left(x_{n}\right)\right)$ and $\beta=\left(\beta\left(x_{1}\right), \beta\left(x_{2}\right), \cdots, \beta\left(x_{n}\right)\right)$ are two fuzzy sets in the universe of discourse $\left(x_{1}, x_{2}, \cdots, x_{n}\right)$. The fuzzy cross-entropy of $\alpha$ from $\beta$ is defined as follows:

$h(\alpha, \beta)=\sum_{j=1}^{n}\left(\alpha\left(x_{j}\right) \ln \frac{\alpha\left(x_{j}\right)}{\frac{1}{2}\left(\alpha\left(x_{j}\right)+\beta\left(x_{j}\right)\right)}+\left(1-\alpha\left(x_{j}\right)\right) \ln \frac{1-\alpha\left(x_{j}\right)}{1-\frac{1}{2}\left(\alpha\left(x_{j}\right)+\beta\left(x_{j}\right)\right)}\right)$,

which indicates the degree of discrimination of $\alpha$ from $\beta$.

However, $h(\alpha, \beta)$ is not symmetric with respect to its arguments. Shang and Jiang (1997) proposed a symmetric discrimination information measure:

$$
I(\alpha, \beta)=h(\alpha, \beta)+h(\beta, \alpha) .
$$

Moreover, there are $I(\alpha, \beta) \geq 0$ and $I(\alpha, \beta)=0$ if and only if $\alpha=\beta$.

Then, the cross-entropy and symmetric discrimination information measures between two fuzzy sets are extended to these measures between PFSs. In order to do so, let us consider two group of picture fuzzy numbers $\alpha=\left(\mu_{\alpha_{j}}, \eta_{\alpha_{j}}, v_{\alpha_{j}}\right)$ and $\beta=\left(\mu_{\beta_{j}}, \eta_{\beta_{j}}, v_{\beta_{j}}\right)$, $j=1,2, \cdots, n$.

Thus based on Eq. (3), the amount of information for discrimination of $\mu_{\alpha_{j}}$ from $\mu_{\beta_{j}}$ $(j=1,2, \cdots, n)$ can be given by:

$$
C^{\mu}(\alpha, \beta, \mu)=\mu_{\alpha_{j}} \ln \frac{\mu_{\alpha_{j}}}{\frac{1}{2}\left(\mu_{\alpha_{j}}+\mu_{\beta_{j}}\right)}+\left(1-\mu_{\alpha_{j}}\right) \ln \frac{1-\mu_{\alpha_{j}}}{1-\frac{1}{2}\left(\mu_{\alpha_{j}}+\mu_{\beta_{j}}\right)}
$$


Therefore, the expected information based on the single degree of positive membership for discrimination of $\alpha$ against $\beta$ is expressed by:

$$
C^{\mu}(\alpha, \beta)=\sum_{j=1}^{n}\left(\mu_{\alpha_{j}} \ln \frac{\mu_{\alpha_{j}}}{\frac{1}{2}\left(\mu_{\alpha_{j}}+\mu_{\beta_{j}}\right)}+\left(1-\mu_{\alpha_{j}}\right) \ln \frac{1-\mu_{\alpha_{j}}}{1-\frac{1}{2}\left(\mu_{\alpha_{j}}+\mu_{\beta_{j}}\right)}\right) .
$$

Similarly, considering the degree of neutral membership and the degree of negative membership, we have the following amounts of information:

$$
\begin{aligned}
& C^{\eta}(\alpha, \beta)=\sum_{j=1}^{n}\left(\eta_{\alpha_{j}} \ln \frac{\eta_{\alpha_{j}}}{\frac{1}{2}\left(\eta_{\alpha_{j}}+\eta_{\beta_{j}}\right)}+\left(1-\eta_{\alpha_{j}}\right) \ln \frac{1-\eta_{\alpha_{j}}}{1-\frac{1}{2}\left(\eta_{\alpha_{j}}+\eta_{\beta_{j}}\right)}\right) \\
& C^{v}(\alpha, \beta)=\sum_{j=1}^{n}\left(v_{\alpha_{j}} \ln \frac{v_{\alpha_{j}}}{\frac{1}{2}\left(v_{\alpha_{j}}+v_{\beta_{j}}\right)}+\left(1-v_{\alpha_{j}}\right) \ln \frac{1-v_{\alpha_{j}}}{1-\frac{1}{2}\left(v_{\alpha_{j}}+v_{\beta_{j}}\right)}\right)
\end{aligned}
$$

Hence, a novel picture fuzzy cross-entropy measure between $\alpha$ and $\beta$ is obtained as the sum of the three amounts:

$$
\begin{aligned}
C(\alpha, \beta)= & \sum_{j=1}^{n}\left(\mu_{\alpha_{j}} \ln \frac{\mu_{\alpha_{j}}}{\frac{1}{2}\left(\mu_{\alpha_{j}}+\mu_{\beta_{j}}\right)}+\left(1-\mu_{\alpha_{j}}\right) \ln \frac{1-\mu_{\alpha_{j}}}{1-\frac{1}{2}\left(\mu_{\alpha_{j}}+\mu_{\beta_{j}}\right)}\right)+ \\
& \sum_{j=1}^{n}\left(\eta_{\alpha_{j}} \ln \frac{\eta_{\alpha_{j}}}{\frac{1}{2}\left(\eta_{\alpha_{j}}+\eta_{\beta_{j}}\right)}+\left(1-\eta_{\alpha_{j}}\right) \ln \frac{1-\eta_{\alpha_{j}}}{1-\frac{1}{2}\left(\eta_{\alpha_{j}}+\eta_{\beta_{j}}\right)}\right)+ \\
& \sum_{j=1}^{n}\left(v_{\alpha_{j}} \ln \frac{v_{\alpha_{j}}}{\frac{1}{2}\left(v_{\alpha_{j}}+v_{\beta_{j}}\right)}+\left(1-v_{\alpha_{j}}\right) \ln \frac{1-v_{\alpha_{j}}}{1-\frac{1}{2}\left(v_{\alpha_{j}}+v_{\beta_{j}}\right)}\right),
\end{aligned}
$$

which also indicates discrimination degree of $\alpha$ from $\beta$. According to Shannon's (1948) inequality, one can easily prove that $C(\alpha, \beta) \geq 0$ and $C(\alpha, \beta)=0$ if and only if $\alpha=\beta$. Then, $C(\alpha, \beta)$ is not symmetric. So it should be modified to a symmetric discrimination information measure for PFSs as:

$$
D(\alpha, \beta)=C(\alpha, \beta)+C(\beta, \alpha) .
$$

The larger the difference between $\alpha$ and $\beta$ is, the larger $D(\alpha, \beta)$ is. 
If we consider the weights of $\alpha$ and $\beta$, a picture fuzzy weighted cross-entropy measure between $\alpha$ and $\beta$ is proposed as follows:

$$
\begin{aligned}
C_{\omega}(\alpha, \beta)= & \sum_{j=1}^{n} \omega_{j}\left(\mu_{\alpha_{j}} \ln \frac{\mu_{\alpha_{j}}}{\frac{1}{2}\left(\mu_{\alpha_{j}}+\mu_{\beta_{j}}\right)}+\left(1-\mu_{\alpha_{j}}\right) \ln \frac{1-\mu_{\alpha_{j}}}{1-\frac{1}{2}\left(\mu_{\alpha_{j}}+\mu_{\beta_{j}}\right)}\right)+ \\
& \sum_{j=1}^{n} \omega_{j}\left(\eta_{\alpha_{j}} \ln \frac{\eta_{\alpha_{j}}}{\frac{1}{2}\left(\eta_{\alpha_{j}}+\eta_{\beta_{j}}\right)}+\left(1-\eta_{\alpha_{j}}\right) \ln \frac{1-\eta_{\alpha_{j}}}{1-\frac{1}{2}\left(\eta_{\alpha_{j}}+\eta_{\beta_{j}}\right)}\right)+ \\
& \sum_{j=1}^{n} \omega_{j}\left(v_{\alpha_{j}} \ln \frac{1}{\frac{1}{2}\left(v_{\alpha_{j}}+v_{\beta_{j}}\right)}+\left(1-v_{\alpha_{j}}\right) \ln \frac{1-v_{\alpha_{j}}}{1-\frac{1}{2}\left(v_{\alpha_{j}}+v_{\beta_{j}}\right)}\right)
\end{aligned}
$$

where $\omega=\left(\omega_{1}, \omega_{2}, \cdots, \omega_{n}\right)^{T}$ is the weight vector of $\alpha, \beta(j=1,2, \cdots, n)$, with $\omega_{j} \in[0,1]$, $j=1,2, \cdots, n, \sum_{j=1}^{n} \omega_{j}=1$,

which also indicates discrimination degree of $\alpha$ from $\beta$. According to Shannon's inequality (5), one can easily prove that $C_{\omega}(\alpha, \beta) \geq 0$ and $C_{\omega}(\alpha, \beta)=0$ if and only if $\alpha=\beta$. Then, $C_{\omega}(\alpha, \beta)$ is not symmetric. So it should be modified to a symmetric discrimination information measure for PFSs as

$$
D_{\omega}(\alpha, \beta)=C_{\omega}(\alpha, \beta)+C_{\omega}(\beta, \alpha) .
$$

The larger the difference between $\alpha$ and $\beta$ is, the larger $D_{\omega}(\alpha, \beta)$ is.

\section{Models for multiple attribute decision making based on cross-entropy with picture fuzzy information}

Based on cross-entropy with picture fuzzy information, in this section, we shall propose the corresponding model for multiple attribute decision making with picture fuzzy information. Let $A=\left\{A_{1}, A_{2}, \cdots, A_{m}\right\}$ be a discrete set of alternatives, and $G=\left\{G_{1}, G_{2}, \cdots, G_{n}\right\}$ be the set of attributes, $\omega=\left(\omega_{1}, \omega_{2}, \cdots, \omega_{n}\right)$ is the weighting vector of the attribute $G_{j}(j=1,2, \cdots, n)$, where $\omega_{j} \in[0,1], \sum_{j=1}^{n} \omega_{j}=1$. Suppose that $\tilde{R}=\left(\tilde{r}_{i j}\right)_{m \times n}=\left(\mu_{i j}, \eta_{i j}, v_{i j}\right)_{m \times n}$ is the picture fuzzy decision matrix, where $\mu_{i j}$ indicates the degree of positive membership that the alternative $A_{i}$ satisfies the attribute $G_{j}$ given by the decision maker, $\eta_{i j}$ indicates the degree of neutral membership that the alternative $A_{i}$ doesn't satisfy the attribute $G_{j}, v_{i j}$ indicates the degree that the alternative $A_{i}$ doesn't satisfy the attribute $G_{j}$ given by the decision maker, $\mu_{i j} \in[0,1], \eta_{i j} \in[0,1], v_{i j} \in[0,1]$, $\mu_{i j}+\eta_{i j}+v_{i j} \leq 1, \pi_{i j}=1-\left(\mu_{i j}+\eta_{i j}+v_{i j}\right) \quad i=1,2, \cdots, m, j=1,2, \cdots, n$. 
In multiple attribute decision-making environments, the concept of ideal point has been used to help identify the best alternative in the decision set. Although the ideal alternative does not exist in real world, it does provide a useful theoretical construct against which to evaluate alternatives. Hence, we can define an ideal attribute value in the ideal alternative $A^{+}$.

Let $\tilde{r}_{j}^{+}=\left(\mu_{j}^{+}, \eta_{j}^{+}, v_{j}^{+}\right)(j=1,2, \cdots, n)$, where

$$
\begin{aligned}
& \mu_{j}^{+}=\max _{i}\left\{\mu_{i j}\right\}, \eta_{j}^{+}=\min _{i}\left\{\eta_{i j}\right\}, v_{j}^{+}=\min _{i}\left\{v_{i j}\right\}, \\
& \pi_{j}^{+}=1-\mu_{j}^{+}-\eta_{j}^{+}-v_{j}^{+}=1-\max _{i}\left\{\mu_{i j}\right\}-\min _{i}\left\{\eta_{i j}\right\}-\min _{i}\left\{v_{i j}\right\}, j=1,2, \cdots, n .
\end{aligned}
$$

Then we call:

$$
A^{+}=\left(\tilde{r}_{1}^{+}, \tilde{r}_{2}^{+}, \cdots, \tilde{r}_{n}^{+}\right),
$$

the relative picture fuzzy ideal alternative.

In the following, we apply the cross-entropy to the MADM problems with hesitant fuzzy information.

Step 1. Define the alternatives $A_{i}$ and $A^{+}$:

$$
\begin{gathered}
A_{i}=\left(\left(\mu_{i 1}, \eta_{i 1}, v_{i 1}\right),\left(\mu_{i 2}, \eta_{i 2}, v_{i 2}\right) \cdots,\left(\mu_{i n}, \eta_{i n}, v_{i n}\right)\right), i=1,2, \cdots, m ; \\
A^{+}=\left(\left(\mu_{1}^{+}, \eta_{1}^{+}, v_{1}^{+}\right),\left(\mu_{2}^{+}, \eta_{2}^{+}, v_{2}^{+}\right) \cdots,\left(\mu_{n}^{+}, \eta_{n}^{+}, v_{n}^{+}\right)\right) ; \\
\mu_{j}^{+}=\max _{i}\left\{\mu_{i j}\right\}, \eta_{j}^{+}=\min _{i}\left\{\eta_{i j}\right\}, v_{j}^{+}=\min _{i}\left\{v_{i j}\right\}, j=1,2, \cdots, n .
\end{gathered}
$$

Step 2. Calculate t picture fuzzy weighted cross-entropy between alternatives $A_{i}$ and $A^{+}$:

$$
\begin{aligned}
C\left(A_{i}, A^{+}\right)= & \sum_{j=1}^{n} \omega_{j}\left(\mu_{i j} \ln \frac{\mu_{i j}}{\frac{1}{2}\left(\mu_{i j}+\mu_{j}^{+}\right)}+\left(1-\mu_{i j}\right) \ln \frac{1-\mu_{i j}}{1-\frac{1}{2}\left(\mu_{i j}+\mu_{j}^{+}\right)}\right)+ \\
& \sum_{j=1}^{n} \omega_{j}\left(\eta_{i j} \ln \frac{\eta_{i j}}{\frac{1}{2}\left(\eta_{i j}+\eta_{j}^{+}\right)}+\left(1-\eta_{i j}\right) \ln \frac{1-\eta_{i j}}{1-\frac{1}{2}\left(\eta_{i j}+\eta_{j}^{+}\right)}\right)+ \\
& \sum_{j=1}^{n} \omega_{j}\left(v_{i j} \ln \frac{v_{i j}}{\frac{1}{2}\left(v_{i j}+v_{j}^{+}\right)}+\left(1-v_{i j}\right) \ln \frac{1-v_{i j}}{1-\frac{1}{2}\left(v_{i j}+v_{j}^{+}\right)}\right) \\
& i=1,2, \cdots, m .
\end{aligned}
$$

Step 3. Rank all the alternatives $A_{i}(i=1,2, \cdots, m)$ and select the best one(s) in accordance with $C\left(A_{i}, A^{+}\right)(i=1,2, \cdots, m)$. The smaller the value of $C\left(A_{i}, A^{+}\right)$is, the better the alternative $A_{i}$ is. In this case, the alternative $A_{i}$ is close to the ideal alternative $A^{+}$. Through the weighted cross-entropy $C\left(A_{i}, A^{+}\right)(i=1,2, \cdots, m)$ between each alternative and the ideal alternative, the ranking order of all alternatives can be determined and the best one can be easily identified as well. 


\section{Numerical example}

Thus, in this section we shall present a numerical example for potential evaluation of emerging technology commercialization with picture fuzzy information in order to illustrate the method proposed in this paper. Let us suppose there is a problem to deal with the potential evaluation of emerging technology commercialization which is classical multiple attribute decision making problems. There is a panel with five possible emerging technology enterprises $A_{i}(i=1,2,3,4,5)$ to select. The experts selects six attribute to evaluate the five possible emerging technology enterprises: 1) $G_{1}$ is the technical advancement; 2) $G_{2}$ is the potential market and market risk; 3) $G_{3}$ is the industrialization infrastructure; 4) $\mathrm{G}_{4}$ is the development of science and technology; 5) $G_{5}$ is the financial conditions; 6) $\mathrm{G}_{6}$ is the employment creation. In order to avoid influence each other, the decision makers are required to evaluate the five possible emerging technology enterprises $A_{i}(i=1,2,3,4,5)$ under the above six attributes and the decision matrix $\tilde{R}=\left(\tilde{r}_{i j}\right)_{5 \times 6}$ is presented in Table 1, where $\tilde{r}_{i j}(i=1,2,3,4,5, j=1,2,3,4,5,6)$ are in the form of PFNs. The weight vector of $x_{i}(i=1,2, \cdots, 6)$ is: $\omega=(0.12,0.25,0.09,0.16,0.20,0.18)^{\mathrm{T}}$.

Table 1 . The picture fuzzy decision matrix

\begin{tabular}{cccccc}
\hline & $A_{1}$ & $A_{2}$ & $A_{3}$ & $A_{4}$ & $A_{5}$ \\
\hline$x_{1}$ & $(0.53,0.33,0.09)$ & $(0.73,0.12,0.08)$ & $(0.91,0.03,0.02)$ & $(0.85,0.09,0.05)$ & $(0.90,0.05,0.02)$ \\
\hline$x_{2}$ & $(0.89,0.08,0.03)$ & $(0.13,0.64,0.21)$ & $(0.07,0.09,0.05)$ & $(0.74,0.16,0.10)$ & $(0.68,0.08,0.21)$ \\
\hline$x_{3}$ & $(0.42,0.35,0.18)$ & $(0.03,0.82,0.13)$ & $(0.04,0.85,0.10)$ & $(0.02,0.89,0.05)$ & $(0.05,0.87,0.06)$ \\
\hline$x_{4}$ & $(0.08,0.89,0.02)$ & $(0.73,0.15,0.08)$ & $(0.68,0.26,0.06)$ & $(0.08,0.84,0.06)$ & $(0.13,0.75,0.09)$ \\
\hline$x_{5}$ & $(0.33,0.51,0.12)$ & $(0.52,0.31,0.16)$ & $(0.15,0.76,0.07)$ & $(0.16,0.71,0.05)$ & $(0.15,0.73,0.08)$ \\
\hline$x_{6}$ & $(0.17,0.53,0.13)$ & $(0.51,0.24,0.21)$ & $(0.31,0.39,0.25)$ & $(0.81,0.15,0.09)$ & $(0.91,0.03,0.05)$ \\
\hline
\end{tabular}

To get the most desirable emerging technology enterprises, the following steps are involved:

Step 1. Based on the Table 1, we denote the five possible emerging technology enterprises $A_{i}(i=1,2,3,4,5)$ by:

$$
\begin{aligned}
& A_{1}=\{(0.53,0.33,0.09),(0.89,0.08,0.03),(0.42,0.35,0.18) \\
&(0.08,0.89,0.02),(0.33,0.51,0.12),(0.17,0.53,0.13)\} ; \\
& A_{2}=\{(0.73,0.12,0.08),(0.13,0.64,0.21),(0.03,0.82,0.13) \\
&(0.73,0.15,0.08),(0.52,0.31,0.16),(0.51,0.24,0.21)\} ; \\
& A_{3}=\{(0.91,0.03,0.02),(0.07,0.09,0.05),(0.04,0.85,0.10) \\
&(0.68,0.26,0.06),(0.15,0.76,0.07),(0.31,0.39,0.25)\} ;
\end{aligned}
$$




$$
\begin{aligned}
& A_{4}=\{(0.85,0.09,0.05),(0.74,0.16,0.10),(0.02,0.89,0.05) \\
& \\
&(0.08,0.84,0.06),(0.16,0.71,0.05),(0.81,0.15,0.09)\} ; \\
& A_{5}=\{(0.90,0.05,0.02),(0.68,0.08,0.21),(0.05,0.87,0.06) \\
&(0.13,0.75,0.09),(0.15,0.73,0.08),(0.91,0.03,0.05)\} .
\end{aligned}
$$

Step 2. Based on the Table 1 and Eqs (15)-(17), we can get the RPFIS $A^{+}$:

$$
\begin{aligned}
& A^{+}=\{(0.91,0.03,0.02),(0.89,0.08,0.03),(0.42,0.35,0.05) \\
& \\
&(0.73,0.15,0.02),(0.52,0.31,0.05),(0.91,0.03,0.05)\} .
\end{aligned}
$$

Step 3. Calculate the cross-entropy $C_{\omega}\left(A_{i}, A^{+}\right)$between $A_{i}(i=1,2,3,4,5)$ and the RPFIS $A^{+}$by using Eq. (18):

$$
\begin{aligned}
& C_{\omega}\left(A_{1}, A^{+}\right)=0.202, C_{\omega}\left(A_{2}, A^{+}\right)=0.192, C_{\omega}\left(A_{3}, A^{+}\right)=0.225, \\
& C_{\omega}\left(A_{4}, A^{+}\right)=0.167, C_{\omega}\left(A_{5}, A^{+}\right)=0.144 .
\end{aligned}
$$

Step 4. Rank the emerging technology enterprises $A_{i}(i=1,2,3,4,5)$ in accordance with the cross-entropy $C_{\omega}\left(A_{i}, A^{+}\right)(i=1,2,3,4,5)$ :

$$
A_{5} \succ A_{4} \succ A_{2} \succ A_{1} \succ A_{3} \text {. }
$$

Thus, the most desirable emerging technology enterprise is $A_{5}$.

\section{Conclusions}

The proposed method differs from previous approaches for fuzzy multiple attribute decision making not only due to the fact that the proposed method use the picture fuzzy set theory, but also due to the consideration of the degree of neutral membership besides degree of positive membership and degree of negative membership in the evaluation of the alternative with respect to attribute, which makes it have more feasible and practical than other traditional decision making methods in real decision making problems. Therefore, its advantage is easily reflecting the ambiguous nature of subjective judgments because the picture fuzzy sets are suitable for capturing imprecise, uncertain, and inconsistent information in the multiple attribute decision making analysis. In this paper, we investigate the multiple attribute decision making problems with picture fuzzy information. The advantage of picture fuzzy set is easily reflecting the ambiguous nature of subjective judgments because the picture fuzzy sets are suitable for capturing imprecise, uncertain, and inconsistent information in the multiple attribute decision making analysis. Thus, the cross entropy of picture fuzzy sets, called picture fuzzy cross entropy, is proposed as an extension of the cross entropy of fuzzy sets. Then, a multiple attribute decision making method based on the proposed picture fuzzy cross entropy is established in which attribute values for alternatives are picture fuzzy numbers. In decision making process, we utilize the picture fuzzy weighted cross entropy between 
the ideal alternative and an alternative to rank the alternatives corresponding to the cross entropy values and to select the most desirable one(s). In the future, we shall investigate picture fuzzy multiple attribute group decision making problems and apply the picture fuzzy cross-entropy to solve practical applications in other areas such as expert system, information fusion system, and medical diagnoses.

\section{Acknowledgments}

The work was supported by the National Natural Science Foundation of China under Grant No. 61174149 and 71571128 and the Humanities and Social Sciences Foundation of Ministry of Education of the People's Republic of China (No.14YJCZH082, 15XJA630006) and the construction plan of scientific research innovation team for colleges and universities in Sichuan Province (15TD0004).

\section{References}

Abdullah, L.; Najib, L. 2016. A new preference scale MCDM method based on interval-valued intuitionistic fuzzy sets and the analytic hierarchy process, Soft Computing 20(2): 511-523. http://dx.doi.org/10.1007/s00500-014-1519-y

Atanassov, K.1986. Intuitionistic fuzzy sets, Fuzzy Sets and Systems 20: 87-96. http://dx.doi.org/10.1016/S0165-0114(86)80034-3

Atanassov, K. 1989. More on intuitionistic fuzzy sets, Fuzzy Sets and Systems 33: 37-46. http://dx.doi.org/10.1016/0165-0114(89)90215-7

Chen, T. Y. 2014. Interval-valued intuitionistic fuzzy QUALIFLEX method with a likelihoodbased comparison approach for multiple criteria decision analysis, Information Sciences 261: 149-169. http://dx.doi.org/10.1016/j.ins.2013.08.054

Cuong, B. 2013. Picture fuzzy sets-first results, part 1, in Seminar "Neuro-Fuzzy Systems with Applications". Institute of Mathematics, Hanoi.

Deluca, A.; Fernuni, S. 1972. A definition of non-probabilistic entropy in the setting of fuzzy sets theory, Information and Control 20: 301-312. http://dx.doi.org/10.1016/S0019-9958(72)90199-4

Kosareva, N.; Krylovas, A. 2013. Comparison of accuracy in ranking alternatives performing generalized fuzzy average functions, Technological and Economic Development of Economy 19(1): 162-187. http://dx.doi.org/10.3846/20294913.2012.763072

Krohling, R. A.; Pacheco, A. G. C.; Siviero, A. L. T. 2013. IF-TODIM: an intuitionistic fuzzy TODIM to multi-criteria decision making, Knowledge-Based Systems 53: 142-146.

http://dx.doi.org/10.1016/j.knosys.2013.08.028

Kullback, S.; Leibler, R. A. 1951. On information and sufficiency, Annals of the Institute of Statistical Mathematics 4: 99-111. http://dx.doi.org/10.1214/aoms/1177729694

Lin, J. 1991. Divergence measures based on Shannon entropy, IEEE Transactions on Information Theory 37: 145-151. http://dx.doi.org/10.1109/18.61115

Lourenzutti, R.; Krohling, R. A. 2013. A study of TODIM in a intuitionistic fuzzy and random environment, Expert Systems with Applications 40(16): 6459-6468.

http://dx.doi.org/10.1016/j.eswa.2013.05.070

Razavi Hajiagha, S. H.; Hashemi, S. S.; Zavadskas, E. K. 2013. A complex proportional assessment method for group decision making in an interval-valued intuitionistic fuzzy environment, Technological and Economic Development of Economy 19(1): 22-37.

http://dx.doi.org/10.3846/20294913.2012.762953 
Razavi Hajiagha, S. H.; Mahdiraji, H. A.; Hashemi, S. A.; Zavadskas, E. K. 2015. Evolving a linear programming technique for MAGDM problems with interval valued intuitionistic fuzzy information, Expert Systems with Applications 42(23): 9318-9325.

http://dx.doi.org/10.1016/j.eswa.2015.07.067

Shang, X. G.; Jiang, W. S. 1997. A note on fuzzy information measures, Pattern Recognition Letters 18: 425-432. http://dx.doi.org/10.1016/S0167-8655(97)00028-7

Shannon, C. E. 1948. A mathematical theory of communication, Bell System Technical Journal 27: 379-423. http://dx.doi.org/10.1002/j.1538-7305.1948.tb01338.x

Singh, P. 2014. Correlation coefficients for picture fuzzy sets, Journal of Intelligent \& Fuzzy Systems 27: 2857-2868.

Son, L. H. 2015. DPFCM: a novel distributed picture fuzzy clustering method on picture fuzzy sets, Expert System with Applications 2: 51-66. http://dx.doi.org/10.1016/j.eswa.2014.07.026

Thong, N. T. 2015. HIFCF: an effective hybrid model between picture fuzzy clustering and intuitionistic fuzzy recommender systems for medical diagnosis, Expert Systems with Applications 42(7): 3682-3701. http://dx.doi.org/10.1016/j.eswa.2014.12.042

Thong, P. H.; Son, L. H. 2015. A new approach to multi-variables fuzzy forecasting using picture fuzzy clustering and picture fuzzy rules interpolation method, in 6th International Conference on Knowledge and Systems Engineering (KSE 2014), 9-10 October 2014, Hanoi, Vietnam, 679-690. http://dx.doi.org/10.1007/978-3-319-11680-8_54

Vlachos, I. K.; Sergiadis, G. D. 2007. Intuitionistic fuzzy information-applications to pattern recognition, Pattern Recognition Letters 28: 197-206. http://dx.doi.org/10.1016/j.patrec.2006.07.004

Wang, W. Z.; Liu, X. W. 2014. Some hesitant fuzzy geometric operators and their application to multiple attribute group decision making, Technological and Economic Development of Economy 20(3): 371-390. http://dx.doi.org/10.3846/20294913.2013.877094

Wu, J. 2015. A SD-IITFOWA operator and TOPSIS based approach for MAGDM problems with intuitionistic trapezoidal fuzzy numbers, Technological and Economic Development of Economy 21(1): 28-47. http://dx.doi.org/10.3846/20294913.2014.946982

Wu, J.; Huang, H. B.; Cao, Q. W. 2013. Research on AHP with interval-valued intuitionistic fuzzy sets and its application in multi-criteria decision making problems, Applied Mathematical Modelling 37(24): 9898-9906. http://dx.doi.org/10.1016/j.apm.2013.05.035

Xia, M. M.; Xu, Z. S. 2012. Entropy/cross entropy-based group decision making under intuitionistic fuzzy environment, Information Fusion 13: 31-47.

http://dx.doi.org/10.1016/j.inffus.2010.12.001

Xu, Z. S.; Xia, M. M. 2012. Hesitant fuzzy entropy and cross-entropy and their use in multiattribute decision-making, International Journal of Intelligent Systems 27: 799-822.

http://dx.doi.org/10.1002/int.21548

Ye, J. 2009a. Fault diagnosis of turbine based on fuzzy cross entropy of vague sets, Expert Systems with Applications 36: 8103-8106. http://dx.doi.org/10.1016/j.eswa.2008.10.017

Ye, J. 2009b. Multicriteria fuzzy decision-making method based on the intuitionistic fuzzy crossentropy, in Y. C. Tang, J. Lawry, V. N. Huynh (Eds.). Proceedings in International Conference on Intelligent Human-Machine Systems and Cybernetics, 1: IEEE Computer Society, 59-61. http://dx.doi.org/10.1109/ihmsc.2009.23

Ye, J. 2011. Fuzzy cross entropy of interval-valued intuitionistic fuzzy sets and its optimal decision-making method based on the weights of alternatives, Expert Systems with Applications 38: 6179-6183. http://dx.doi.org/10.1016/j.eswa.2010.11.052

Zadeh, L. A. 1965. Fuzzy sets, Information Control 8: 338-353.

http://dx.doi.org/10.1016/S0019-9958(65)90241-X 
Zadeh, L. A. 1968. Probability measures of fuzzy events, Journal of Mathematical Analysis and Applications 23: 421-427. http://dx.doi.org/10.1016/0022-247X(68)90078-4

Zavadskas, E. K.; Antucheviciene, J.; Razavi Hajiagha, S. H.; Hashemi, S. S. 2014. Extension of weighted aggregated sum product assessment with interval-valued intuitionistic fuzzy numbers (WASPAS-IVIF), Applied Soft Computing 24: 1013-1021.

http://dx.doi.org/10.1016/j.asoc.2014.08.031

Zhang, Q. S.; Jiang, S. Y. 2008. A note on information entropy measures for vague sets, Information Sciences 178: 4184-4191. http://dx.doi.org/10.1016/j.ins.2008.07.003

Zhang, Q. S.; Jiang, S. Y. 2010. Relationships between entropy and similarity measure of intervalvalued intuitionistic fuzzy sets, International Journal of Intelligent Systems 25: 1121-1140.

Guiwu WEI has an MSc and a PhD degree in applied mathematics from SouthWest Petroleum University, Business Administration from School of Economics and Management at SouthWest Jiaotong University, China, respectively. From May 2010 to April 2012, he was a Postdoctoral Researcher with the School of Economics and Management, Tsinghua University, Beijing, China. He is a Professor in the School of Business at Sichuan Normal University. He has published more than 90 papers in journals, books and conference proceedings including journals such as Omega, Decision Support Systems, Expert Systems with Applications, Applied Soft Computing, Knowledge and Information Systems, Computers \& Industrial Engineering, Knowledge-based Systems, International Journal of Uncertainty, Fuzziness and Knowledge-Based Systems, International Journal of Computational Intelligence Systems and Information: An International Interdisciplinary Journal. He has published 1 book. He has participated in several scientific committees and serves as a reviewer in a wide range of journals including Computers \& Industrial Engineering, International Journal of Information Technology and Decision Making, Knowledge-based Systems, Information Sciences, International Journal of Computational Intelligence Systems and European Journal of Operational Research. He is currently interested in Aggregation Operators, Decision Making and Computing with Words. 